JOURNAL OF

FUNCTION SPACES AND APPLICATIONS

Volume 5, Number 1 (2007), 9-26 (c) 2007, Scientific Horizon

http://www.jfsa.net

\title{
Initial value problems for first order impulsive integro-differential equations of Volterra type in Banach spaces
}

\author{
Jiang Zhu*, Yajuan Yu and Vasile Postolică \\ (Communicated by George Isac)
}

2000 Mathematics Subject Classification. 45J05, 47H07, 34A12.

Keywords and phrases. Impulsive integro-differential equations, ordered Banach spaces, noncompactness measure.

\footnotetext{
Abstract. In this paper, we use a new method and combining the partial ordering method to study the existence of the solutions for the first order nonlinear impulsive integro-differential equations of Volterra type on finite interval in Banach spaces and for the first order nonlinear impulsive integro-differential equations of Volterra type on infinite interval with infinite number impulsive times in Banach spaces. By introducing an interim space and using progressive estimation method, some restrictive conditions on impulsive terms, used before, such as, prior estimation, noncompactness measure estimations are deleted.
}

*The first author is supported by natural science foundation of the EDJP and JSQLGC, China. 


\section{Introduction}

Impulsive differential equations arise naturally from a wide variety of applications, such as spacecraft control, inspection processes in operations research, drug administration, and threshold theory in biology, see [1], [8] and [10]. Initial value problems for first order impulsive integro-differential equations of Volterra type in Banach Spaces has been studied in [5], [6] and [9]. However, to obtain the solutions, some restricted conditions on impulsive terms, such as, prior estimation, noncompactness measure estimation, are needed in [5], [6] and [9]. In this paper, we use a new method and combining the partial ordering method to study the existence of the solutions for the first order nonlinear impulsive integro-differential equations of Volterra type on infinite interval with infinite number impulsive times in Banach spaces. By introducing an interim space and using progressive estimation method, the restrictive conditions on impulsive terms, such as, prior estimation, noncompactness measure estimations are deleted. Our results and methods are different from the ones in [5], [6] and [9].

Let $E$ be a Banach space, $P \subset E$ be a closed convex cone in $E$. The partial order $\leq$ in $E$ is induced by $P$, that is $x \leq y$, if and only if $y-x \in P$. $P$ is said to be normal if there exists a positive constant $K$, such that $\theta \leq x \leq y$ implies $\|x\| \leq K\|y\|$, where $\theta$ denotes the zero element of $E$, and the smallest $K$ is called the normal constant of $P . P$ is said to be regular if $x_{1} \leq x_{2} \leq \cdots \leq y$ implies $\left\|x_{n}-x\right\| \rightarrow 0$ as $n \rightarrow \infty$ for some $x \in E$. For details on cone theory, see [6].

In this paper, we consider the following initial value problems for the first order nonlinear impulsive integro-differential equations of Volterra type on infinite interval with infinite number impulsive times in Banach spaces.

$$
\left\{\begin{array}{l}
u^{\prime}=f(t, u, T u), t \in J, t \neq t_{i}, \\
\left.\triangle u\right|_{t=t_{i}}=I_{i}\left(u\left(t_{i}^{-}\right)\right),(i=1,2, \cdots), \\
u(0)=u_{0},
\end{array}\right.
$$

where $J=[0, \infty), u_{0} \in E, f \in C[J \times E \times E, E]$,

$$
(T u)(t)=\int_{0}^{t} k(t, s) u(s) d s, t \in J,
$$

$k \in C\left[D, R_{+}\right]$and $D=\{(t, s) \in J \times J: t \geq s\} . I_{i} \in C[E, E]$ $(i=1,2,3, \cdots), 0<t_{1}<\cdots<t_{i}<\cdots, t_{i} \rightarrow \infty,\left.\quad \Delta u\right|_{t=t_{i}}$ denotes the jump of $x(t)$ at $t=t_{k}$, that is,

$$
\left.\Delta u\right|_{t=t_{i}}=u\left(t_{i}^{+}\right)-u\left(t_{i}^{-}\right),
$$


where $u\left(t_{k}^{+}\right)$and $u\left(t_{k}^{-}\right)$denote the right and left limits of $u(t)$ at $t=t_{k}$, respectively.

Let

$$
\begin{gathered}
J_{0}=\left[0, t_{1}\right], \quad J_{m}=\left(t_{m}, t_{m+1}\right], m=1,2,3, \cdots, \\
\bar{J}_{m}=\left[t_{m}, t_{m+1}\right], m=1,2,3, \cdots
\end{gathered}
$$

Let

$$
k_{i}=\max \left\{k(t, s):(t, s) \in \bar{J}_{i} \times \bar{J}_{i}\right\}, i=0,1,2, \cdots .
$$

Let $J^{\prime}=J \backslash\left\{t_{1}, \cdots, t_{m}, \cdots\right\}$. Let $P C[J, E]=\{u: u$ is a map of from $J \rightarrow E$ such that $u(t)$ is continuous at $t \neq t_{i}$, left continuous at $t=t_{i}$ and $u\left(t_{i}^{+}\right)$exists, $\left.i=1,2,3, \cdots\right\}, \triangle t_{i}=t_{i+1}-t_{i}$. Then $u \in P C[J, E] \cap C^{1}\left[J^{\prime}, E\right]$ is called a solution of the initial value problem (1) if it satisfies (1).

For fixed $u_{i} \in C\left[\bar{J}_{i}, E\right], i=0,1,2, \cdots, k$, we introduce the interim spaces as follows:

$$
\begin{aligned}
& P C\left[J_{0, k}, u_{0}, \cdots, u_{k}, E\right] \\
& =\left\{u(t) \in P C\left[J_{0, k}, E\right]:\left.u(t)\right|_{J_{i}}=u_{i}(t), i=0,1, \cdots, k-1\right\},
\end{aligned}
$$

where $J_{0, k}=\bigcup_{i=0}^{k} J_{i}, k \geq 1$. Let $J_{0, k}^{\prime}=J_{0, k} \backslash\left\{t_{1}, \cdots, t_{k}\right\}, k \geq 1$, the meaning of $P C\left[J_{0, k}, E\right]$ is same as $P C[J, E]$.

\section{Main Results}

In this section, we discuss the existences of the solutions for the initial value problem (1) and need the following comparison result.

Lemma 1. Let $p \in P C\left[J_{0, i}, E\right] \cap C^{1}\left[J_{0, i}^{\prime}, E\right]$ (where $J_{0,0}=J_{0}$ ) be such that

$$
\left\{\begin{array}{l}
p^{\prime} \leq-M p-N T_{i} p, \quad t \in J_{i}, \\
p\left(t_{i}^{+}\right) \leq \theta, \\
p(t)=\theta, \text { if } i \geq 1, t \in J_{0, i-1},
\end{array}\right.
$$

where $M \geq 0, N \geq 0$ are constants, and

$$
T_{i} p(t)=\int_{t_{i}}^{t} k(t, s) p(s) d s, t \in J_{i} .
$$

If

$$
\left\{\begin{array}{l}
N k_{i} \triangle t_{i}\left(e^{M \triangle t_{i}}-1\right) \leq M, \text { if } M>0, \\
N k_{i}\left(\triangle t_{i}\right)^{2} \leq 1, \text { if } M=0 .
\end{array}\right.
$$


Then, $p(t) \leq \theta, t \in J_{i}$.

The proof of Lemma 1 is similar to the proof of Lemma 4.1.1 in [6] or refer to [3].

We give the following conditions for the next results:

$\left(H_{1}\right)$ There exist $v_{0}, w_{0} \in P C[J, E] \cap C^{1}\left[J^{\prime}, E\right]$ such that $v_{0}(t) \leq w_{0}(t)$ $(t \in J)$ and

$$
\begin{aligned}
& v_{0}^{\prime} \leq f\left(t, v_{0}, T v_{0}\right), \quad t \in J, \quad t \neq t_{i}, \\
& \left.\Delta v_{0}\right|_{t=t_{i}} \leq I_{i}\left(v_{0}\left(t_{i}\right)\right) \quad(i=1,2,3, \cdots), v_{0}(0) \leq u_{0}, \\
& w_{0}^{\prime} \geq f\left(t, w_{0}, T w_{0}\right), \quad t \in J, \quad t \neq t_{i}, \\
& \left.\Delta w_{0}\right|_{t=t_{i}} \geq I_{i}\left(w_{0}\left(t_{i}\right)\right) \quad(i=1,2,3, \cdots), w_{0}(0) \geq u_{0} .
\end{aligned}
$$

$\left(H_{2}\right)$ There exist constants $M \geq 0$ and $N \geq 0$ such that

$$
f(t, u, v)-f(t, \bar{u}, \bar{v}) \geq-M(u-\bar{u})-N(v-\bar{v}), \quad t \in J,
$$

whenever $v_{0}(t) \leq \bar{u} \leq u \leq w_{0}(t), \quad\left(T v_{0}\right)(t) \leq \bar{v} \leq v \leq\left(T w_{0}\right)(t)$.

$\left(H_{3}\right)$ For any $i=1,2,3, \cdots$

$$
I_{i}(u)-I_{i}(\bar{u}) \geq-(u-\bar{u}),
$$

whenever $v_{0}\left(t_{i}\right) \leq \bar{u} \leq u \leq w_{0}\left(t_{i}\right)$.

$\left(H_{4}\right)$ For any $r>0$, and any $i=1,2,3, \cdots$, there exist constants $c_{r, i} \geq 0$ and $c_{r, i}^{*} \geq 0$ such that

$$
\alpha\left(f\left(\bar{J}_{i}, B, B^{*}\right)\right) \leq c_{r, i} \alpha(B)+c_{r, i}^{*} \alpha\left(B^{*}\right), \quad B, B^{*} \subset B_{r},
$$

where $B_{r}=\{u \in E:\|u\| \leq r\}$ and $\alpha$ denotes the Kuratowski measure of noncompactness in $E$.

Let $\left[v_{0}, w_{0}\right]=\left\{u \in P C[J, E]: u_{0}(t) \leq u(t) \leq w_{0}(t), \quad t \in J\right\}$.

Theorem 2. Let $P$ be normal and the conditions $\left(H_{1}\right) \sim\left(H_{4}\right)$ be satisfied. Assume that the inequality (5) holds. Then the initial value problem (1) has at least a solution $u \in P C[J, E] \cap C^{1}\left[J^{\prime}, E\right]$ in $\left[v_{0}, w_{0}\right]$.

Proof. Let $\left[v_{0}, w_{0}\right]_{0}=\left\{u \in C\left[J_{0}, E\right]: u_{0}(t) \leq u(t) \leq w_{0}(t), \quad t \in J_{0}\right\}$. It follows from conditions $\left(H_{1}\right),\left(H_{2}\right),\left(H_{4}\right)$ and by using Theorem 4.1.1 in [6] or Theorem 3.1.1 in [5] that initial value problem

$$
\begin{gathered}
u^{\prime}=f(t, u, T u), t \in J_{0}, \\
u(0)=u_{0},
\end{gathered}
$$


has a minimal solution and a maximal solution $\bar{u}_{0}, u_{0}^{*} \in\left[v_{0}, w_{0}\right]_{0}$, respectively. By (2)

$$
P C\left[J_{0,1}, \bar{u}_{0}, E\right]=\left\{u(t) \in P C\left[J_{0,1}, E\right]:\left.u(t)\right|_{J_{0}}=\bar{u}_{0}(t)\right\} .
$$

Let $\left[v_{0}, w_{0}\right]_{1}=\left\{u \in P C\left[J_{0,1}, \bar{u}_{0}, E\right]: v_{0}(t) \leq u(t) \leq w_{0}(t), \quad t \in J_{1}\right\}$.

For any $\eta \in\left[v_{0}, w_{0}\right]_{1}$, we consider the initial value problem of linear integro-differential equation in $E$ :

$$
u^{\prime}+M u=-N T u+g(t), \quad t \in J_{1}, u\left(t_{1}^{+}\right)=\bar{u}_{0}\left(t_{1}^{-}\right)+I_{1}\left(\bar{u}_{0}\left(t_{1}^{-}\right)\right),
$$

where

$$
g(t)=f(t, \eta(t),(T \eta)(t))+M \eta(t)+N(T \eta)(t) .
$$

It is easy to show that $u \in P C\left[J_{0,1}, \bar{u}_{0}, E\right]$ is a solution of the linear initial value problem (6) if and only if $u \in P C\left[J_{0,1}, \bar{u}_{0}, E\right]$ is a solution of the following integral equation:

(8) $u(t)=e^{-M\left(t-t_{1}\right)}\left\{u\left(t_{1}^{+}\right)+\int_{t_{1}}^{t}[g(s)-N(T u)(s)] e^{M\left(s-t_{1}\right)} d s\right\}, \quad t \in J_{1}$.

Since

$$
\begin{aligned}
\int_{t_{1}}^{t} N(T u)(s) e^{M(s-t)} d s= & \int_{t_{1}}^{t} \int_{0}^{t_{1}} N e^{M(s-t)} k(s, r) \bar{u}_{0}(r) d r d s \\
& +\int_{t_{1}}^{t} N\left(\int_{s}^{t} e^{M(r-t)} k(r, s) d r\right) u(s) d s .
\end{aligned}
$$

(8) can be written as follows:

$$
u(t)=F(t)-\int_{t_{1}}^{t} K_{1}(t, s) u(s) d s, \quad t \in J_{1},
$$

where

$$
\begin{aligned}
F(t)= & e^{-M\left(t-t_{1}\right)}\left\{u\left(t_{1}^{+}\right)+\int_{t_{1}}^{t} g(s) e^{M\left(s-t_{1}\right)} d s\right\} \\
& \left.+\int_{t_{1}}^{t} \int_{0}^{t_{1}} N e^{M(s-t)} k(s, r) \bar{u}_{0}(r) d r d s\right\}, \\
K_{1}(t, s)= & N e^{-M t} \int_{s}^{t} e^{M r} k(r, s) d r, \quad t_{1} \leq s \leq t \leq t_{2} .
\end{aligned}
$$


Clearly, the integral equation (9) is a linear Volterra equation in $E$. Thus, by Theorem 1.4.2 in [5], it has a unique solution $v$ in $C\left[\bar{J}_{1}, E\right]$. Letting

$$
u(t)=\left\{\begin{array}{l}
\bar{u}_{0}(t), \text { if } t \in J_{0} \\
v(t), \text { if } t \in J_{1} .
\end{array}\right.
$$

Therefore, $u \in P C\left[J_{0,1}, \bar{u}_{0}, E\right]$ is the unique solution of the initial value problems (6). Letting $u=A \eta$, then the operator $A$ maps $\left[v_{0}, w_{0}\right]_{1}$ into $P C\left[J_{0,1}, \bar{u}_{0}, E\right]$.

Now, we prove that

(i) $v_{0} \leq A v_{0}$ and $A w_{0} \leq w_{0}, t \in J_{0,1}$,

(ii) $A$ is increasing in $\left[v_{0}, w_{0}\right]_{1}$.

If $t \in J_{0}$, for any $\eta \in\left[v_{0}, w_{0}\right]_{1}, A \eta(t)=\bar{u}_{0}(t)$, thus, (i) and (ii) hold. If $t \in J_{1}$, let $v_{1}=A v_{0}$ and $p=v_{0}-v_{1}$. Then, from (6), we have

$$
\begin{aligned}
& v_{1}^{\prime}+M v_{1}=-N T v_{1}+f\left(t, v_{0}, T v_{0}\right)+M v_{0}+N T v_{0}, \quad t \in J_{1}, \\
& v_{1}\left(t_{1}^{+}\right)=\bar{u}_{0}\left(t_{1}^{-}\right)+I_{1}\left(\bar{u}_{0}\left(t_{1}^{-}\right)\right),
\end{aligned}
$$

and so, from the condition $\left(H_{1}\right),\left(H_{3}\right)$, it follows that

$$
\begin{aligned}
p^{\prime} & \leq-M p-N T p, \quad t \in J_{1}, \\
p\left(t_{1}^{+}\right) & =v_{0}\left(t_{1}^{+}\right)-v_{1}\left(t_{1}^{+}\right) \\
& \leq v_{0}\left(t_{1}^{-}\right)+I_{1}\left(v_{0}\left(t_{1}^{-}\right)\right)-\bar{u}_{0}\left(t_{1}^{-}\right)-I_{1}\left(\bar{u}_{0}\left(t_{1}^{-}\right)\right) \\
& \leq \bar{u}_{0}\left(t_{1}^{-}\right)+I_{1}\left(\bar{u}_{0}\left(t_{1}^{-}\right)\right)-\bar{u}_{0}\left(t_{1}^{-}\right)-I_{1}\left(\bar{u}_{0}\left(t_{1}^{-}\right)\right) \\
& =\theta .
\end{aligned}
$$

By the definition of $P C\left[J_{0,1}, \bar{u}_{0}, E\right]$, we have

$$
\begin{aligned}
p^{\prime} & \leq-M p-N T p \\
& =-M p-N \int_{0}^{t} k(t, s) p(s) d s \\
& =-M p-N \int_{0}^{t_{1}} k(t, s) p(s) d s-N \int_{t_{1}}^{t} k(t, s) p(s) d s \\
& =-M p-N \int_{t_{1}}^{t} k(t, s) p(s) d s \\
& =-M p-N T_{1} p, t \in J_{1}, \\
p(t) & =\theta, t \in J_{0} .
\end{aligned}
$$

Thus, by Lemma 1 , it follows that $p(t) \leq \theta$ for all $t \in J_{1}$, that is, $v_{0} \leq A v_{0}$, for $t \in J_{1}$. Similarly, we can prove that $A w_{0} \leq w_{0}, t \in J_{1}$. Let 
$\eta_{1}, \eta_{2} \in\left[v_{0}, w_{0}\right]_{1}$ with $\eta_{1} \leq \eta_{2}$ and $p=u_{1}-u_{2}$, where $u_{1}=A \eta_{1}$ and $u_{2}=A \eta_{2}$. From (6), it follows that

$$
\begin{aligned}
& u_{i}^{\prime}+M u_{i}=-N T u_{i}+f\left(t, \eta_{i}, T \eta_{i}\right)+M \eta_{i}+N T \eta_{i}, \quad t \in J_{1}, \\
& u_{i}\left(t_{1}^{+}\right)=\bar{u}_{0}\left(t_{1}^{-}\right)+I_{1}\left(\bar{u}_{0}\left(t_{1}^{-}\right)\right), i=1,2,
\end{aligned}
$$

and so, by using the condition $\left(\mathrm{H}_{2}\right)$, we can show that

$$
\begin{aligned}
p^{\prime} & \leq-M p-N T p, \quad t \in J_{1}, \\
p\left(t_{1}^{+}\right) & =u_{1}\left(t_{1}^{+}\right)-u_{2}\left(t_{1}^{+}\right) \\
& =u_{1}\left(t_{1}^{-}\right)+I_{1}\left(u_{1}\left(t_{1}^{-}\right)\right)-u_{2}\left(t_{1}^{-}\right)-I_{1}\left(u_{2}\left(t_{1}^{-}\right)\right) \\
& =\bar{u}_{0}\left(t_{1}^{-}\right)+I_{1}\left(\bar{u}_{0}\left(t_{1}^{-}\right)\right)-\bar{u}_{0}\left(t_{1}^{-}\right)-I_{1}\left(\bar{u}_{0}\left(t_{1}^{-}\right)\right) \\
& =\theta .
\end{aligned}
$$

By the definition of $P C\left[J_{0,1}, \bar{u}_{0}, E\right]$, we have

$$
\begin{aligned}
p^{\prime} & \leq-M p-N T p \\
& =-M p-N \int_{0}^{t} k(t, s) p(s) d s \\
& =-M p-N \int_{0}^{t_{1}} k(t, s) p(s) d s-N \int_{t_{1}}^{t} k(t, s) p(s) d s \\
& =-M p-N \int_{t_{1}}^{t} k(t, s) p(s) d s \\
& =-M p-N T_{1} p, t \in J_{1}, \\
p(t) & =\theta, t \in J_{0} .
\end{aligned}
$$

Therefore, by Lemma 1 , it follows that $p(t) \leq \theta$ for all $t \in J_{1}$, that is, $A \eta_{1} \leq A \eta_{2}$, for all $t \in J_{1}$ and so (ii) is proved.

Let

$$
v_{n}^{1}=A v_{n-1}^{1}, \quad w_{n}^{1}=A w_{n-1}^{1} \quad(n=1,2,3, \cdots),
$$

where $v_{0}^{1}=v_{0}, w_{0}^{1}=w_{0}$. By the conclusions (i) and (ii), we have (10)

$$
v_{0}(t) \leq v_{1}^{1}(t) \leq \cdots \leq v_{n}^{1}(t) \leq \cdots \leq w_{n}^{1}(t) \leq \cdots \leq w_{1}^{1}(t) \leq w_{0}(t), \quad t \in J_{0,1} .
$$

Then, by the normality of $P$, the set $V=\left\{v_{n}^{1}: n=1,2, \cdots\right\}$ is a bounded set in $C\left[\bar{J}_{1}, E\right]$, here, for $v_{n}^{1} \in C\left[\bar{J}_{1}, E\right]$, we understand that $v_{n}^{1}\left(t_{1}\right)=v_{n}^{1}\left(t_{1}^{+}\right)$. Since the condition $\left(\mathrm{H}_{4}\right)$ implies that the set $f\left(\bar{J}_{1}, B_{r}, B_{r}\right)$ is bounded for any $r>0$, So, there exists a constant $c_{0}>0$ such that (11)

$$
\begin{gathered}
\left\|f\left(t, v_{n-1}(t),\left(T v_{n-1}\right)(t)\right)+M v_{n-1}(t)-N\left(T\left(v_{n}-v_{n-1}\right)\right)(t)\right\| \leq c_{0}, \\
t \in \bar{J}_{1} \quad(n=1,2,3, \cdots) .
\end{gathered}
$$


By the definition of $v_{n}^{1}$ and (8), we have

$$
\begin{aligned}
v_{n}^{1}(t)= & e^{-M\left(t-t_{1}\right)}\left(u\left(t_{1}^{+}\right)+\int_{t_{1}}^{t}\left[f\left(s, v_{n-1}^{1}(s),\left(T v_{n-1}^{1}\right)(s)\right)+M v_{n-1}^{1}(s)\right.\right. \\
& \left.\left.-N\left(T\left(v_{n}^{1}-v_{n-1}^{1}\right)\right)(s)\right] e^{M\left(s-t_{1}\right)} d s\right), \quad t \in J_{1}(n=1,2,3 \cdots) .
\end{aligned}
$$

Then, from (11) and (12), all the functions in $V$ is equicontinuous on $\bar{J}_{1}$ and so by Theorem 1.2.2 in [6], the function $m(t)=\alpha(V(t))$ is continuous on $\bar{J}_{1}$, where $V(t)=\left\{v_{n}^{1}(t): n=1,2, \cdots\right\}$ for a fixed $t \in \bar{J}_{1}$. Applying Corollary 1.2 .1 in $[6]$ to $(12)$, we have

$$
\begin{aligned}
m(t) \leq & 2 \int_{t_{1}}^{t} \alpha\left(\left\{e ^ { - M ( t - s ) } \left[f\left(s, v_{n-1}^{1}(s),\left(T v_{n-1}^{1}\right)(s)\right)\right.\right.\right. \\
& \left.\left.\left.+M v_{n-1}^{1}(s)-N\left(T\left(v_{n}^{1}-v_{n-1}^{1}\right)\right)(s)\right]: n=1,2, \cdots\right\}\right) d s \\
\leq & 2 \int_{t_{1}}^{t}[\alpha(f(s, V(s),(T V)(s))+M \alpha(V(s))+2 N \alpha(T V)(s))] d s
\end{aligned}
$$

where $(T V)(t)=\left\{\left(T v_{n}^{1}\right)(t): n=1,2, \cdots\right\}$. On the other hand, by Corollary 1.2.1 in [6], we have

$$
\begin{aligned}
\alpha((T V)(t)) & \leq 2 \int_{0}^{t} \alpha\left(\left\{k(t, s) v_{n}^{1}(s): n=0,1,2, \cdots\right\}\right) d s \\
& \leq k_{1} 2 \int_{t_{1}}^{t} m(s) d s, \quad t \in J_{1} .
\end{aligned}
$$

Thus, from the condition $\left(\mathrm{H}_{4}\right)$, there exists constant $c \geq 0$ and $c^{*} \geq 0$ such that

$$
\alpha\left(f \left(t, V(t),(T V(t)) \leq c \alpha(V(t))+c^{*} \alpha((T V)(t)), \quad t \in \bar{J}_{1} .\right.\right.
$$

Therefore, from $(13) \sim(15)$, it follows that

$$
\begin{aligned}
m(t) & \leq 2(c+M) \int_{t_{1}}^{t} m(s) d s+4 k_{1}\left(c^{*}+2 N\right) \int_{t_{1}}^{t} d s \int_{t_{1}}^{s} m\left(s^{\prime}\right) d s^{\prime} \\
& =2(c+M) \int_{t_{1}}^{t} m(s) d s+4 k_{1}\left(c^{*}+2 N\right) \int_{t_{1}}^{t}(t-s) m(s) d s \\
& \leq \tau \int_{t_{1}}^{t} m(s) d s, \quad t \in \bar{J}_{1},
\end{aligned}
$$


where $\tau=2(c+M)+4 k_{1} \Delta t_{1}\left(c^{*}+2 N\right)=$ const $\geq 0$. Let

$$
y(t)=\int_{t_{1}}^{t} m(s) d s, \quad z(t)=y(t) e^{-\tau t}, \quad t \in \bar{J}_{1} .
$$

Then, from (16), we have

$$
z^{\prime}(t)=\left[y^{\prime}(t)-\tau y(t)\right] e^{-\tau t}=\left[m(t)-\tau \int_{t_{1}}^{t} m(s) d s\right] e^{-\tau t} \leq 0, \quad t \in \bar{J}_{1}
$$

and so $z(t) \leq z\left(t_{1}\right)=y\left(t_{1}\right) e^{-\tau t_{1}}=0$ for all $t \in \bar{J}_{1}$. Thus $z(t) \equiv 0$ for all $t \in \bar{J}_{1}$, which implies that $m(t) \equiv 0$ for all $t \in \bar{J}_{1}$ and, by Theorem 1.2.5 in [6], $V$ is a relatively compact set in $C\left[\bar{J}_{1}, E\right]$. Thus, $\left\{v_{n}^{1}\right\}$ has a convergent subsequence in $C\left[\bar{J}_{1}, E\right]$, suppose that $\left\{v_{n_{i}}^{1}\right\}$ converges to $\bar{u}^{1}$ in $C\left[\bar{J}_{1}, E\right]$. Since $\left\{v_{n}^{1}\right\}$ is increasing, for any positive integer $n$, we have $v_{n_{i}}^{1} \geq v_{n}^{1}$, whenever $n_{i} \geq n$. Taking $n_{i} \rightarrow \infty$, we get that $\bar{u}^{1} \geq v_{n}^{1}$. Since $P$ is normal, from inequality

$$
\theta \leq \bar{u}^{1}-v_{n}^{1} \leq \bar{u}^{1}-v_{n_{i}}^{1}, \forall n \geq n_{i},
$$

we have

$$
\left\|\bar{u}^{1}-v_{n}^{1}\right\| \leq K\left\|\bar{u}^{1}-v_{n_{i}}^{1}\right\| \forall n \geq n .
$$

Since $\lim _{n \rightarrow \infty}\left\|\bar{u}^{1}-v_{n_{i}}^{1}\right\|=0$, we obtain $\lim _{n \rightarrow \infty}\left\|\bar{u}^{1}-v_{n}^{1}\right\|=0$. Thus, $\left\{v_{n}^{1}\right\}$ converges to $\bar{u}^{1}$ in $C\left[\bar{J}_{1}, E\right]$, that is, the sequence $\left\{v_{n}^{1}\right\}$ converges uniformly to $\bar{u}^{1} \in C\left[\bar{J}_{1}, E\right]$ on $\bar{J}_{1}$. Since $v_{n}^{1} \in\left[v_{0}, w_{0}\right]_{1}$, we have $\left.v_{n}^{1}\right|_{J_{0}}=\bar{u}_{0}$, thus $\left\{v_{n}^{1}\right\}$ converges uniformly to $\bar{u}_{0}$ on $J_{0}$. Let

$$
\bar{v}_{1}(t)= \begin{cases}\bar{u}_{0}(t), & t \in J_{0} . \\ \bar{u}^{1}(t), & t \in J_{1} .\end{cases}
$$

Then, $\left\{v_{n}^{1}\right\}$ converges uniformly to $\bar{v}_{1}$ on $J_{0,1}$.

Now, we have

$$
\begin{aligned}
& f\left(t, v_{n-1}^{1},\left(T v_{n-1}^{1}\right)(t)\right)+M v_{n-1}^{1}(t)-N\left(T\left(v_{n}^{1}-v_{n-1}^{1}\right)\right)(t) \\
& \rightarrow f\left(t, \bar{v}_{1}(t),\left(T \bar{v}_{1}\right)(t)\right)+M \bar{v}_{1}(t) \quad(n \rightarrow \infty), \quad t \in \bar{J}_{1} .
\end{aligned}
$$

Further, from (11), it follows that

$$
\begin{aligned}
& \| f\left(t, v_{n-1}^{1}(t),\left(T v_{n-1}^{1}\right)(t)\right)+M v_{n-1}^{1}(t)-N\left(T\left(v_{n}^{1}-v_{n-1}^{1}\right)\right)(t) \\
& -f\left(t, \bar{v}_{1}(t),\left(T \bar{v}_{1}\right)(t)\right)-M \bar{v}_{1}(t) \| \\
& \quad \leq 2 c_{0}, \quad t \in \bar{J}_{1}(n=1,2,3, \cdots)
\end{aligned}
$$


and so, from (17) and (18), by taking limits in both sides of (12), we get

$$
\begin{aligned}
\bar{v}_{1}(t)= & e^{-M\left(t-t_{1}\right)}\left\{u\left(t_{1}^{+}\right)+\int_{t_{1}}^{t}\left[f\left(s, \bar{v}_{1}(s),\left(T \bar{v}_{1}\right)(s)\right)\right.\right. \\
& \left.\left.+M \bar{v}_{1}(s)\right] e^{M\left(s-t_{1}\right)} d s\right\}, \quad t \in \bar{J}_{1} .
\end{aligned}
$$

Hence we can easily show that $\bar{v}_{1} \in P C\left[J_{0,1}, E\right] \cap C^{1}\left[J_{0,1}^{\prime}, E\right]$ and $\bar{v}_{1}$ is a solution of the initial value problem

$$
\left\{\begin{array}{l}
u^{\prime}(t)=f(t, u, T u), \\
u\left(t_{1}^{+}\right)=\bar{u}_{0}\left(t_{1}^{-}\right)+I_{1}\left(\bar{u}_{0}\left(t_{1}^{-}\right)\right), \\
u(0)=u_{0}
\end{array} \quad t \in J_{0,1}, t \neq t_{1},\right.
$$

That is, $\bar{v}_{1}(t)$ is a solution of the initial value problem (1) on $J_{0,1}$.

Assume that we have get a solution $\bar{v}_{m-1}(t)$ of $(1)$ on $J_{0, m-1}$ in $\left[v_{0}, w_{0}\right]_{m-1}$, such that $\bar{v}_{m-1}(t)$ satisfies

$$
\bar{v}_{m-1}(t)= \begin{cases}\bar{u}_{0}(t), & t \in J_{0}, \\ \bar{u}^{1}(t), & t \in J_{1}, \\ \cdots & \\ \bar{u}^{m-1}(t), & t \in J_{m-1},\end{cases}
$$

and

$$
\begin{cases}u^{\prime}(t)=f(t, u, T u), t \in J_{0, m-1}, & t \neq t_{k}, \\ u\left(t_{k}^{+}\right)=\bar{u}^{k-1}\left(t_{k}^{-}\right)+I_{k}\left(\bar{u}^{k-1}\left(t_{1}^{-}\right)\right), & \\ u(0)=u_{0}, & k=1,2, \cdot \cdot, m-1 .\end{cases}
$$

where $\bar{u}^{k}(t)$, is a uniformly limitation of the increasing sequence $\left\{v_{n}^{k}\right\} \subset$ $\left[v_{0}, w_{0}\right]_{k}$ on $J_{k}, k=1,2, \cdot \cdot m-1$. Let

$\left[v_{0}, w_{0}\right]_{m}$

$=\left\{u \in P C\left[J_{0, m}, \bar{u}_{0}, \bar{u}^{1}(t), \cdots, \bar{u}^{m-1}(t), E\right]: v_{0}(t) \leq u(t) \leq w_{0}(t), t \in J_{m}\right\}$.

It is similar to the above proof of (10), we can get an increasing sequence $v_{0}(t) \leq v_{1}^{m}(t) \leq \cdots \leq v_{n}^{m}(t) \leq \cdots \leq w_{n}^{m}(t) \leq \cdots \leq w_{1}^{m}(t) \leq w_{0}(t), t \in J_{0, m}$. 
in $\left[v_{0}, w_{0}\right]_{m}$ and $\left\{v_{n}^{m}(t)\right\}$ uniformly converges to $\bar{u}^{m}(t)$ on $J_{m}$. If we let

$$
\bar{v}_{m}(t)=\left\{\begin{array}{l}
\bar{u}_{0}(t), t \in J_{0}, \\
\bar{u}^{1}(t), t \in J_{1}, \\
\cdots \\
\bar{u}^{m-1}(t), t \in J_{m-1}, \\
\bar{u}^{m}(t), t \in J_{m} .
\end{array}\right.
$$

Then, by the similar proof of (19), we have

$$
\begin{aligned}
\bar{v}_{m}(t)= & e^{-M\left(t-t_{m}\right)}\left\{\bar{u}^{m-1}\left(t_{m}^{-}\right)+I\left(\bar{u}^{m-1}\left(t_{m}^{-}\right)\right)\right. \\
& \left.+\int_{t_{m}}^{t}\left[f\left(s, \bar{v}_{m}(s),\left(T \bar{v}_{m}\right)(s)\right)+M \bar{v}_{m}(s)\right] e^{M\left(s-t_{m}\right)} d s\right\}, \quad t \in J_{m} .
\end{aligned}
$$

That is, $\bar{v}_{m}(t)$ is a solution of the following

$$
\left\{\begin{array}{l}
u^{\prime}(t)=f(t, u, T u), \quad t \in J_{m}, \\
u\left(t_{m}^{+}\right)=\bar{u}^{m-1}\left(t_{m}^{-}\right)+I_{m}\left(\bar{u}^{m-1}\left(t_{m}^{-}\right)\right),
\end{array}\right.
$$

Then $\bar{v}_{m}(t)$ is a solution of

$$
\begin{cases}u^{\prime}(t)=f(t, u, T u), t \in J_{0, m}, & t \neq t_{k}, \\ u\left(t_{k}^{+}\right)=\bar{u}^{k-1}\left(t_{k}^{-}\right)+I_{k}\left(\bar{u}^{k-1}\left(t_{k}^{-}\right)\right), & \\ u(0)=u_{0}, & k=1,2, \cdot \cdot, m,\end{cases}
$$

thus, $\bar{v}_{m}(t)$ satisfies

$$
\begin{cases}\bar{v}_{m}^{\prime}(t)=f\left(t, \bar{v}_{m}, T \bar{v}_{m}\right), & t \in J_{0, m}, t \neq t_{k}, \\ \triangle \bar{v}_{m}\left(t_{k}^{+}\right)=I_{k}\left(\bar{v}_{m}\left(t_{k}^{-}\right)\right), & \\ \bar{v}_{m}(0)=u_{0}, & k=1,2, \cdot \cdot m .\end{cases}
$$

By induction, we can get two sequences $\left\{\bar{v}_{m}(t)\right\} \subset\left[v_{0}, w_{0}\right]_{m}$ and $\left\{\bar{u}^{m}(t)\right)$ such that

$$
\bar{v}_{m}(t)= \begin{cases}\bar{u}_{0}(t), & t \in J_{0}, \\ \bar{u}^{1}(t), & t \in J_{1}, \\ \cdots & \\ \bar{u}^{m-1}(t), & t \in J_{m-1}, \\ \bar{u}^{m}(t), & t \in J_{m} .\end{cases}
$$


and $\bar{v}_{m}(t)$ is a solution of

$$
\begin{cases}u^{\prime}(t)=f(t, u, T u), & t \in J_{0, m}, t \neq t_{k}, \\ u\left(t_{k}^{+}\right)=\bar{u}^{k-1}\left(t_{k}^{-}\right)+I_{k}\left(\bar{u}^{k-1}\left(t_{k}^{-}\right)\right), & \\ u(0)=u_{0}, & k=1,2, \cdot \cdot m, m\end{cases}
$$

that is, $\bar{v}_{m}(t)$ satisfies

$$
\begin{cases}\bar{v}_{m}^{\prime}(t)=f\left(t, \bar{v}_{m}, T \bar{v}_{m}\right), & t \in J_{0, m}, t \neq t_{k}, \\ \triangle \bar{v}_{m}\left(t_{k}^{+}\right)=I_{k}\left(\bar{v}_{m}\left(t_{k}^{-}\right)\right), & \\ \bar{v}_{m}(0)=u_{0}, & k=1,2, \cdot \cdot m,\end{cases}
$$

Let

$$
\bar{v}(t)=\bar{u}^{m}(t), t \in J_{m}, m=0,1,2, \cdots
$$

From $(20),(21)$ and $(22)$ we can know that $\bar{v}(t)$ satisfies

$$
\begin{cases}\bar{v}^{\prime}(t)=f(t, \bar{v}, T \bar{v}), & t \in J, t \neq t_{k}, \\ \triangle \bar{v}\left(t_{k}^{+}\right)=I_{k}\left(\bar{v}\left(t_{k}^{-}\right)\right), & \\ \bar{v}(0)=u_{0}, & k=1,2, \cdots\end{cases}
$$

that is, $\bar{v}(t)$ is a solution of $(1)$ and $\bar{v} \in P C[J, E] \cap C^{1}\left[J^{\prime}, E\right] \cap\left[v_{0}, w_{0}\right]$. The proof is completed.

Remark 1. From the proof of Theorem 2 we can see that if $\bar{u}_{0}$ is different from $u_{0}^{*}$ on $J_{0}$, then the initial value problem (1) has at least two solutions in $\left[v_{0}, w_{0}\right]$.

Remark 2. Condition $\left(H_{4}\right)$ is an improtant, most in use and general condition in nonolinear problems in abstract spaces. Concerening the checking and using of this kind of conditions, one can refer to [2],[4],[6] and $[7]$.

Theorem 3. Let $P$ be regular and the conditions $\left(H_{1}\right) \sim\left(H_{3}\right)$ be satisfied. Assume that the inequality (5) holds. Then the initial value problem (1) has at least a solution $u \in P C[J, E] \cap C^{1}\left[J^{\prime}, E\right]$ in $\left[v_{0}, w_{0}\right]$.

Proof. The proof is similar to that of Theorem 2. The only difference of the proof is that the conclusion $m(t)=\alpha(V(t))=0$ for all $t \in \bar{J}_{1}$ follows directly from the regularity of $P$ and the fact that $\left\{v_{n}^{1}(t)\right\}$ is an increasing sequence. This complete the proof.

From the proof of Theorem 2 we can get the following corollary. 
Corollary 4. If $J=[0, a]$ and $0<t_{1}<\cdots<t_{m}<a$. Let $P$ be normal and the conditions $\left(H_{1}\right) \sim\left(H_{4}\right)$ be satisfied for $i=1,2, \cdots, m$. Assume that the inequality (5) holds. Then the initial value problem (1) has at least a solution $u \in P C[J, E] \cap C^{1}\left[J^{\prime}, E\right]$ in $\left[v_{0}, w_{0}\right]$.

\section{Example}

We consider the following initial value problem of infinite system for the scalar first order impulsive integro-differential equation:

$$
\begin{cases}u_{n}^{\prime}=\frac{1}{3 n}\left[\left(t-u_{n}\right)^{3}+u_{n+1}^{4}\right]-\frac{1}{2} \int_{0}^{t} e^{-t s} u_{n}(s) d s & \\ +\frac{1}{\sqrt{n}}\left(\int_{0}^{t} e^{-t s} u_{2 n}(s) d s\right)^{2}, & 0 \leq t \leq 1, \quad t \neq \frac{1}{2}, \\ \left.\Delta u_{n}\right|_{t=\frac{1}{2}}=-u_{n}\left(\frac{1}{2}\right), & u_{n}(0)=0 \quad(n=1,2,3, \cdots) .\end{cases}
$$

Evidently, $u_{n}(t) \equiv 0 \quad(n=1,2,3, \cdots)$ is not a solution of the initial value problem (23).

Conclusion. The initial value problem (23) has at least a continuously differentiable solutions defined on $\left[0, \frac{1}{2}\right) \cup\left(\frac{1}{2}, 1\right]$ with

$$
0 \leq u_{n}(t) \leq \begin{cases}\frac{t}{n}+\frac{1}{12 n}, & 0 \leq t \leq \frac{1}{2} \\ \leq \frac{t}{n}, \frac{1}{2}<t \leq 1, & (n=1,2,3, \cdots)\end{cases}
$$

In fact, let

$$
a=1, \quad E=c_{0}=\left\{u=\left(u_{1}, u_{2}, \cdots, u_{n}, \cdots\right): u_{n} \rightarrow 0\right\}
$$

with the norm be defined by $\|u\|=\sup _{n}\left|u_{n}\right|$ and

$$
P=\left\{u=\left(u_{1}, u_{2}, \cdots, u_{n}, \cdots\right) \in c_{0}: u_{n} \geq 0, \quad n=1,2,3, \cdots\right\} .
$$

Then $P$ is a normal cone in $E$ and (23) can be regarded as an initial value problem of form (1) in $E$, where

$$
\begin{aligned}
u_{0} & =(0, \cdots, 0, \cdots), \quad J=[0,1], \quad k(t, s)=e^{-t s}, \\
u & =\left(u_{1}, u_{2}, \cdots, u_{n}, \cdots\right), \quad v=\left(v_{1}, v_{2}, \cdots, v_{n}, \cdots\right), \\
f & =\left(f_{1}, f_{2}, \cdots, f_{n}, \cdots\right)
\end{aligned}
$$


with

$$
\begin{gathered}
f_{n}(t, u, v)=\frac{1}{3 n}\left[\left(t-u_{n}\right)^{3}+u_{n+1}^{4}\right]-\frac{1}{2} v_{n}+\frac{1}{\sqrt{n}} v_{2 n}^{2} \\
m=1, t_{1}=\frac{1}{2}, \quad I_{1}(u)=-u .
\end{gathered}
$$

Clearly, $f \in C[J \times E \times E, E]$ and $I_{1} \in C[E, E]$. Let

$$
\begin{aligned}
& v_{0}(t)=(0, \cdots, 0, \cdots), \quad t \in J \\
& w_{0}(t)= \begin{cases}\left(t+\frac{1}{12}, \cdots, \frac{t}{n}+\frac{1}{12 n}, \cdots\right), & 0 \leq t \leq \frac{1}{2}, \\
\left(t, \cdots, \frac{t}{n}, \cdots\right), & \frac{1}{2}<t \leq 1 .\end{cases}
\end{aligned}
$$

Then we have

$$
v_{0}, w_{0} \in P C[J, E] \cap C^{1}\left[J^{\prime}, E\right] \quad\left(J^{\prime}=J \backslash\left\{\frac{1}{2}\right\}=\left[0, \frac{1}{2}\right) \cup\left(\frac{1}{2}, 1\right]\right)
$$

and $v_{0}(t) \leq w_{0}(t)$ for all $t \in J$ and so

$$
\begin{aligned}
v_{0}(0) & =w_{0}(0)=(0, \cdots, 0, \cdots), \\
\left.\Delta v_{0}\right|_{t=\frac{1}{2}} & =(0, \cdots, 0, \cdots)=-v_{0}\left(\frac{1}{2}\right), \\
\left.\Delta w_{0}\right|_{t=\frac{1}{2}} & =\left(-\frac{3}{12}, \cdots,-\frac{3}{12 n}, \cdots\right) \geq-w_{0}\left(\frac{1}{2}\right), \\
v_{0}^{\prime}(t) & =(0, \cdots, 0, \cdots), \quad t \in J, \\
w_{0}^{\prime}(t) & =\left(1, \cdots, \frac{1}{n}, \cdots\right), \quad t \in J, \quad t \neq \frac{1}{2}, \\
f_{n}\left(t, v_{0}(t),\left(T v_{0}\right)(t)\right) & =\frac{t^{3}}{3 n} \geq 0, \quad t \in J \quad(n=1,2,3, \cdots) .
\end{aligned}
$$


In addition, if $0 \leq t \leq \frac{1}{2}$, then we have

$$
\begin{aligned}
f_{n}\left(t, w_{0}(t),\left(T w_{0}\right)(t)\right)= & \frac{1}{3 n}\left[\left(t-\frac{t}{n}-\frac{1}{12 n}\right)^{3}+\left(\frac{t}{n+1}+\frac{1}{12 n+12}\right)^{4}\right] \\
& -\frac{1}{2}\left(\int_{0}^{\frac{1}{2}} e^{-t s}\left(\frac{s}{n}+\frac{1}{12 n}\right) d s\right) \\
& +\frac{1}{\sqrt{n}}\left[\int_{0}^{\frac{1}{2}} e^{-t s}\left(\frac{s}{2 n}+\frac{1}{24 n}\right) d s\right]^{2} \\
\leq & \frac{1}{3 n}\left[\frac{1}{8}+\left(\frac{7}{12(n+1)}\right)^{4}\right] \\
& +\frac{1}{\sqrt{n}}\left[\int_{0}^{t} e^{-t s}\left(\frac{s}{2 n}+\frac{1}{24 n}\right) d s\right]^{2} \\
\leq & \frac{1}{3 n}\left[\frac{1}{8}+\left(\frac{7}{12(n+1)}\right)^{4}\right]+\frac{1}{4 n^{2} \sqrt{n}} \frac{12}{77} \\
\leq & \frac{1}{3 n} \cdot \frac{1}{2}+\frac{1}{4 n^{2}} \cdot \frac{12}{77}<\frac{1}{n} \quad(n=1,2,3, \cdots) .
\end{aligned}
$$

If $\frac{1}{2}<t \leq 1$, we have

$$
\begin{aligned}
f_{n}\left(t, w_{0}(t),\left(T w_{0}\right)(t)\right)= & \frac{1}{3 n}\left[\left(t-\frac{t}{n}\right)^{3}+\left(\frac{t}{n+1}\right)^{4}\right] \\
& -\frac{1}{2 n}\left[\int_{0}^{\frac{1}{2}}\left(s+\frac{1}{12}\right) e^{-t s} d s+\int_{\frac{1}{2}}^{t} e^{-t s} s d s\right] \\
& +\frac{1}{\sqrt{n}}\left(\int_{0}^{\frac{1}{2}} e^{-t s}\left(\frac{s}{2 n}+\frac{1}{24}\right) d s+\int_{\frac{1}{2}}^{t} e^{-t s} \frac{s}{n} d s\right) \\
\leq & \frac{1}{3 n}-\frac{1}{6 n}\left[\int_{0}^{\frac{1}{2}}\left(s+\frac{1}{12}\right) d s+\int_{\frac{1}{2}}^{t} s d s\right] \\
& +\frac{1}{\sqrt{n}}\left(\int_{0}^{\frac{1}{2}}\left(\frac{s}{2 n}+\frac{1}{24}\right) d s+\int_{\frac{1}{2}}^{t} \frac{s}{n} d s\right)^{2} \\
\leq & \frac{1}{3 n}-\frac{7}{48 n}+\frac{1}{6 n^{2}}<\frac{1}{n} \quad(n=1,2,3, \cdots) .
\end{aligned}
$$


Thus $v_{0}$ and $w_{0}$ satisfy the condition $\left(H_{1}\right)$. On the other hand, if $t \in J$. $v_{0}(t) \leq \bar{u} \leq u \leq w_{0}(t)$ and $\left(T v_{0}\right)(t) \leq \bar{v} \leq v \leq\left(T w_{0}\right)(t)$, then we have

$$
\begin{aligned}
0 & \leq \overline{u_{n}} \leq u_{n} \\
& \leq \begin{cases}\frac{t}{n}+\frac{1}{12 n}, & 0 \leq t \leq \frac{1}{2}, \\
\frac{t}{n}, & \frac{1}{2}<t \leq 1,(n=1,2,3, \cdots),\end{cases} \\
0 & \leq \overline{v_{n}} \leq v_{n} \\
& \leq \begin{cases}\frac{1}{2 n}\left(t^{2}+\frac{t}{6}\right) \leq \frac{1}{2 n}\left(t^{2}+\frac{1}{12}\right), & 0 \leq t \leq \frac{1}{2}, \\
\frac{1}{2 n}\left(t^{2}+\frac{1}{12}\right), & \frac{1}{2}<t \leq 1,(n=1,2,3, \cdots),\end{cases}
\end{aligned}
$$

and so, from (24), it follows that

$$
\begin{aligned}
& f_{n}(t, u, v)-f_{n}(t, \bar{u}, \bar{v})= \frac{1}{3 n}\left[\left(t-u_{n}\right)^{3}+u_{n+1}{ }^{4}\right]-\frac{1}{2} v_{n} \\
&+\frac{1}{\sqrt{n}} v_{2 n}^{2}-\frac{1}{3 n}\left[\left(t-\bar{u}_{n}\right)^{3}+\bar{u}_{n+1}^{4}\right]+\frac{1}{2} \bar{v}_{n}-\frac{1}{\sqrt{n}} \bar{v}_{2 n}^{2} \\
& \geq \frac{1}{3 n}\left[\left(t-u_{n}\right)^{3}-\left(t-\bar{u}_{n}\right)^{3}\right]-\frac{1}{2}\left(v_{n}-\bar{v}_{n}\right) \geq-\frac{1}{n}\left(u_{n}-\bar{u}_{n}\right)-\frac{1}{2}\left(v_{n}-\bar{v}_{n}\right) \\
& \geq-\left(u_{n}-\bar{u}_{n}\right)-\frac{1}{2}\left(v_{n}-\bar{v}_{n}\right) \quad(n=1,2,3, \cdots)
\end{aligned}
$$

where we have used the following:

$$
\frac{\partial}{\partial s}(t-s)^{3}=-3(t-s)^{2} \geq-3, \quad 0 \leq s \leq t \leq 1 .
$$

Thus the condition $\left(H_{2}\right)$ is satisfied, where $M=1$ and $N=\frac{1}{2}$. It follows from $(25)$ that $\left(H_{3}\right)$ is satisfied, where $L_{1}=1$. Let $f=g+h$, where $g=\left(g_{1}, g_{2}, \cdots, g_{n}, \cdots\right), h=\left(h_{1}, h_{2}, \cdots, h_{n}, \cdots\right)$ and

$$
g_{n}(t, u, v)=\frac{1}{3 n}\left[\left(t-u_{n}\right)^{3}+u_{n+1}^{4}\right]+\frac{1}{\sqrt{n}} v_{2 n}^{2}, \quad h_{n}(t, u, v)=-\frac{1}{2} v_{n} .
$$

Hence, by imitating the proof of Example 4.1.1 in [6], we can prove that

$$
\alpha\left(g\left(J, B, B^{*}\right)\right)=0, \quad \alpha\left(h\left(J, B, B^{*}\right)\right)=\frac{1}{2} \alpha\left(B^{*}\right)
$$


for any bounded sets $B, B^{*} \subset E$ and so

$$
\alpha\left(f\left(J, B, B^{*}\right)\right) \leq \frac{1}{2} \alpha\left(B^{*}\right)
$$

for any bounded set $B, B^{*} \subset E$. Thus the condition $\left(H_{4}\right)$ is satisfied and, by Corollary 4, the conclusion holds.

Remark 3. Clearly, $\max \{k(t, s):(t, s) \in D\}=1, \max \left\{\triangle t_{i}: i=0,1\right\}=$ $\frac{1}{2}$. Let $l_{0}=\max \{k(t, s):(t, s) \in D\}, \tau=\max \left\{\triangle t_{i}: i=0,1\right\}$ and $M_{0}=M^{-1}\left(e^{M a}-1\right)$, then, we have

$$
\begin{aligned}
M_{0} & =M^{-1}\left(e^{M a}-1\right)=e-1>0, \\
M_{0} e^{-M a} & =M^{-1}\left(1-e^{-M a}\right)=1-e^{-1}>0,
\end{aligned}
$$

and so

$$
\begin{aligned}
N l_{0} M_{0} \tau & =\frac{1}{2}(e-1)>0=\frac{1-L_{1}}{1+1-L_{1}}, \\
N l_{0} M_{0} a e^{-M a}+L_{1} & =\frac{1}{2}\left(1-e^{-1}\right)+1>1 .
\end{aligned}
$$

Therefore, the conclusion of our example can not be obtained from the results of [5], [6] and [9]. This also shows that our results are different from the results in [5], [6] and [9].

\section{References}

[1] D. D. Bainov and P. S. Simeonov, Systems with Impulse Effect, Ellis Horwood, Chichister, 1989.

[2] K. Deimling, Multivalued Differential Equations, De Gruyter, Berlin New York, 1992.

[3] D. Guo, Initial value problems for integro-differential equations of Volterra type in Banach Space, J. Appl. Math. Stochastic. Anal., 7 (1994), 13-23.

[4] D. Guo, Nonlinear Function Analysis, ShanDong Science Press 2002.

[5] D. Guo, V. Lakshmikantham and X. Liu, Nonlinear Integral Equations in Abstract Spaces, Kluwer Academic Publishers, Dorderecht, Boston, London, 1996.

[6] D. Guo, Y. J. Cho and J. Zhu, Partial Ordering Methods in Nonlinear Problems, Nova Science Publishers, Inc. 2004. 
[7] M. Kamenskii, V. Obukhovski and P.Zecca, Condensing Multivalued Maps and Semilinear Differential inclusions in Banach Spaces, Walter de Gruyter, Berlin - New York, 2001.

[8] V. Lakshmikantham, D. D. Bainov and P. S. Simeonov, Theory of Impulsive Differential Equations, World Scientific, Singapore, 1989.

[9] X. Z. Liu and D. Guo, Initial value problems for first order impulsive integro-differential equations in Banach spaces, Comm. Appl. Nonlinear Anal., 2 (1995), 65-83.

[10] A. M. Samoilenko and N.A. Perestyuk, Impulsive Differential Equations, World Scientific, Singapore, 1995.

Department of Mathematics

Xuzhou Normal University

Xuzhou 221116

People's Republic of China

(E-mail : jzhuccy@yahoo.com.cn)

Department of Information and Science

JiangSu Polytechnic

University Changzhou 213016

People's Republic of China

(E-mail : yyjxye@jpu.edu.cn)

Department of Mathematics

Faculty of Sciences

Romanian Academy of Scientists

Bacău State University

Romania

(E-mail : vpostolica@ambra.ro)

(Received : September 2005) 


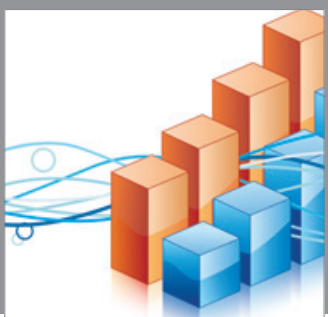

Advances in

Operations Research

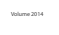

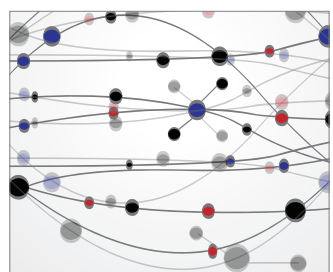

\section{The Scientific} World Journal
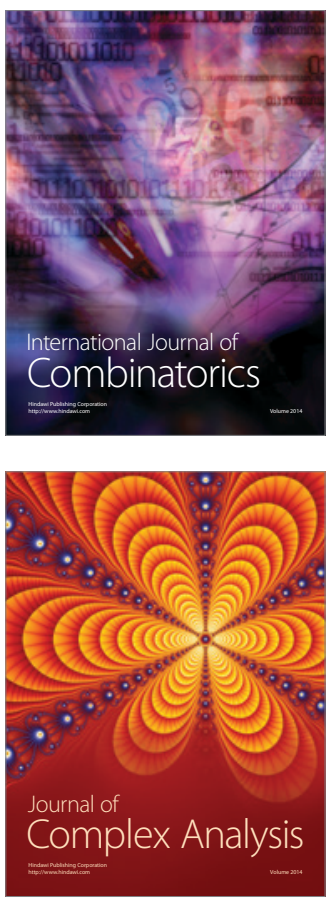

International Journal of

Mathematics and

Mathematical

Sciences
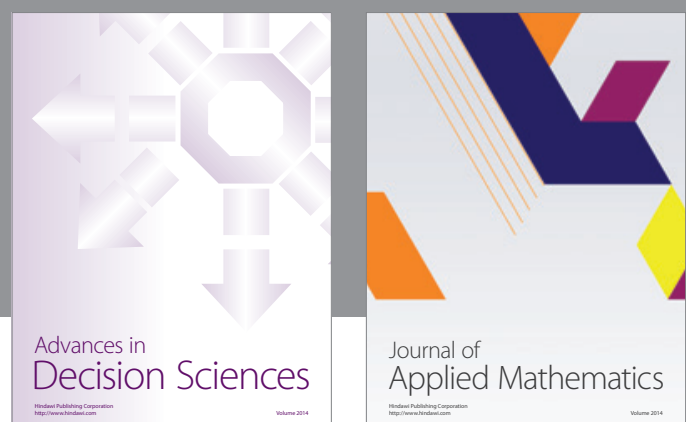

Journal of

Applied Mathematics
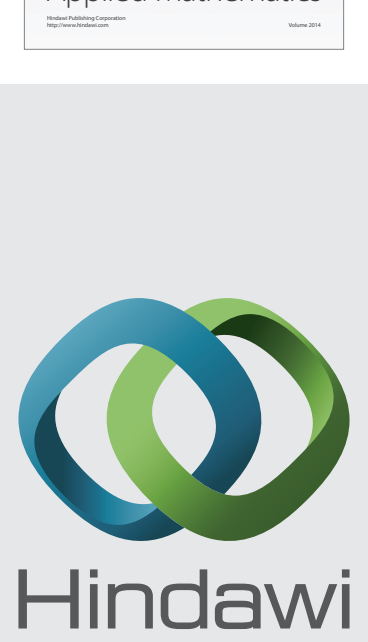

Submit your manuscripts at http://www.hindawi.com
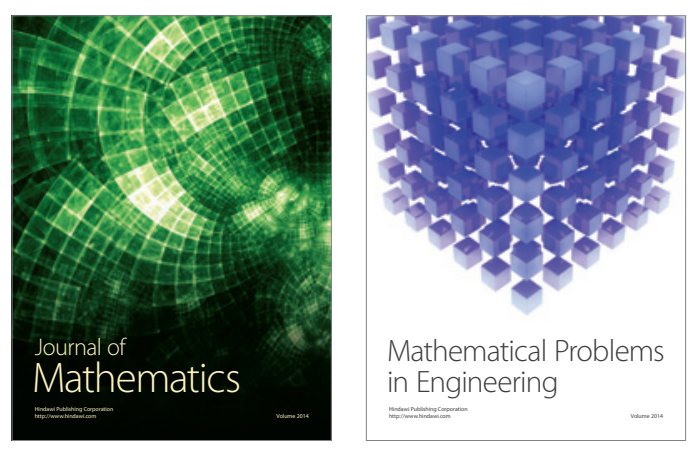

Mathematical Problems in Engineering
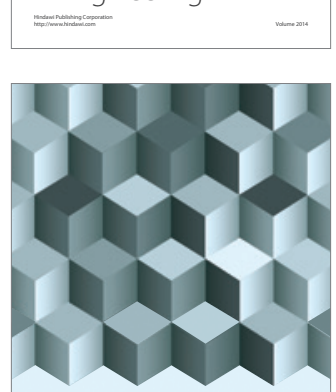

Journal of

Function Spaces
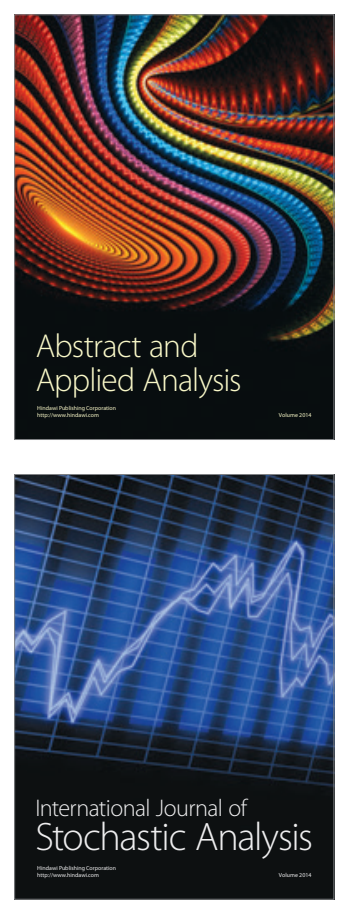

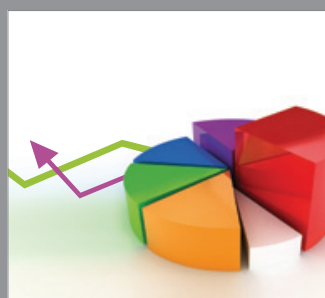

ournal of

Probability and Statistics

Promensencen
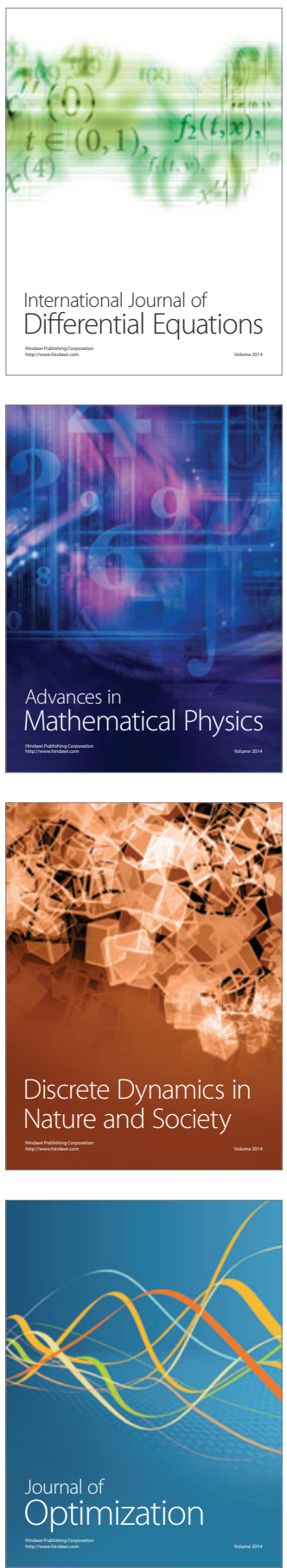